\title{
Concept Study of On-Instrument Wavefront Sensors (OIWFS) for the TMT IRIS Science Instrument
}

\author{
David Loop $^{1, \text { a }}$, Murray Fletcher ${ }^{1}$, Vlad Reshetov ${ }^{1}$, Robert Wooff ${ }^{1}$, Jennifer Dunn ${ }^{1}$, Glen Herriot $^{1}$, \\ Anna Moore ${ }^{2}$, Richard Dekany ${ }^{2}$, Alex Delacroix ${ }^{2}$, Roger Smith $^{2}$, David Hale ${ }^{2}$, James Larkin ${ }^{3}$, Luc \\ Simard $^{4}$, David Crampton ${ }^{4}$, Brent Ellerbroek ${ }^{4}$, Corinne Boyer ${ }^{4}$, and Lianqi Wang ${ }^{4}$ \\ 1 NRC Herzberg Institute of Astrophysics, 5071 W. Saanich Rd., Victoria, BC V9E 2E7, CANADA \\ 2 Caltech Astronomy Department, 1200 E. California Blvd, Pasadena, CA 91125, USA \\ 3 UCLA Infrared Laboratory, 430 Portola Plaza, Los Angeles, CA 90095-1547, USA \\ 4 TMT Observatory, 2632 E. Washington Blvd, Pasadena, CA 91107, USA
}

\begin{abstract}
We report on the concept study of the IRIS On-Instrument WaveFront Sensors (OIWFS), a collaborative effort by NRC-HIA, Caltech, and TMT AO and Instrument teams. Our concept study includes work on the system design of the low order natural guide star wavefront sensors located on-instrument within IRIS as well as the NFIRAOS-IRIS interface. The OIWFS study involves patrol geometry, detector alternatives, sky coverage modeling, acquisition, guiding, and dithering scenarios, and interfaces with NFIRAOS, IRIS, and the TMT observatory. It also includes work on probe optics, mechanics, and controls, detector arrays and controllers, instrument rotator and cable wrap, and thermal and structural design of the OIWFS enclosure.
\end{abstract}

\section{Introduction}

The TMT InfraRed Imaging Spectrograph (IRIS) science instrument is located behind the Narrow Field Infrared Adaptive Optics System (NFIRAOS), a facility multi-conjugate adaptive optics system that provides turbulence compensation over a moderately large field of view ( 2 arcmin) and feeds up to three science instruments. The reference design of NFIRAOS includes one or more tip/tilt/focus natural guide star wavefront sensors within each instrument. NFIRAOS will sharpen the images of natural guide stars and improve the sky coverage for tip/tilt sensing with these on-instrument wavefront sensors (OIWFS).

To maximize sky coverage, these on-instrument wavefront sensors will sense natural guide stars in the infrared. For the IRIS science instrument, two tip/tilt wavefront sensors will be equipped to perform fast guiding, and one tip/tilt/focus wavefront sensor will be equipped to calibrate the focus biases in the Laser Guide Star WFS induced by the variations in the range to the sodium layer. The IRIS OIWFS will also enable computation of blind modes, comprised of a mix of focus/astigmatism and tilt anisoplanatism modes.

The key requirements for the IRIS OIWFS are derived from the TMT science requirements and decisions made in the NFIRAOS reference design.

- 50 percent sky coverage at the Galactic Pole

- 2 tip/tilt and 1 tip/tilt/focus wavefront sensors, for fast guiding, focus, and tilt anisoplanatism

- 2 mas positioning accuracy at delivered focal plane

- High acquisition probability = low acquisition time

- 2 mas alignment between NFIRAOS delivered beam and IRIS science detector through instrument rotator

\section{Concept Down Select}

The first part of the concept study explored architectural alternatives for the natural guide star wavefront sensors, and the following object select mechanisms were considered.

\footnotetext{
a e-mail: david.loop@nrc-cnrc.gc.ca
}

This is an Open Access article distributed under the terms of the Creative Commons Attribution-Noncommercial License, which permits unrestricted use, distribution, and reproduction in any noncommercial medium, provided the original work is properly cited. 


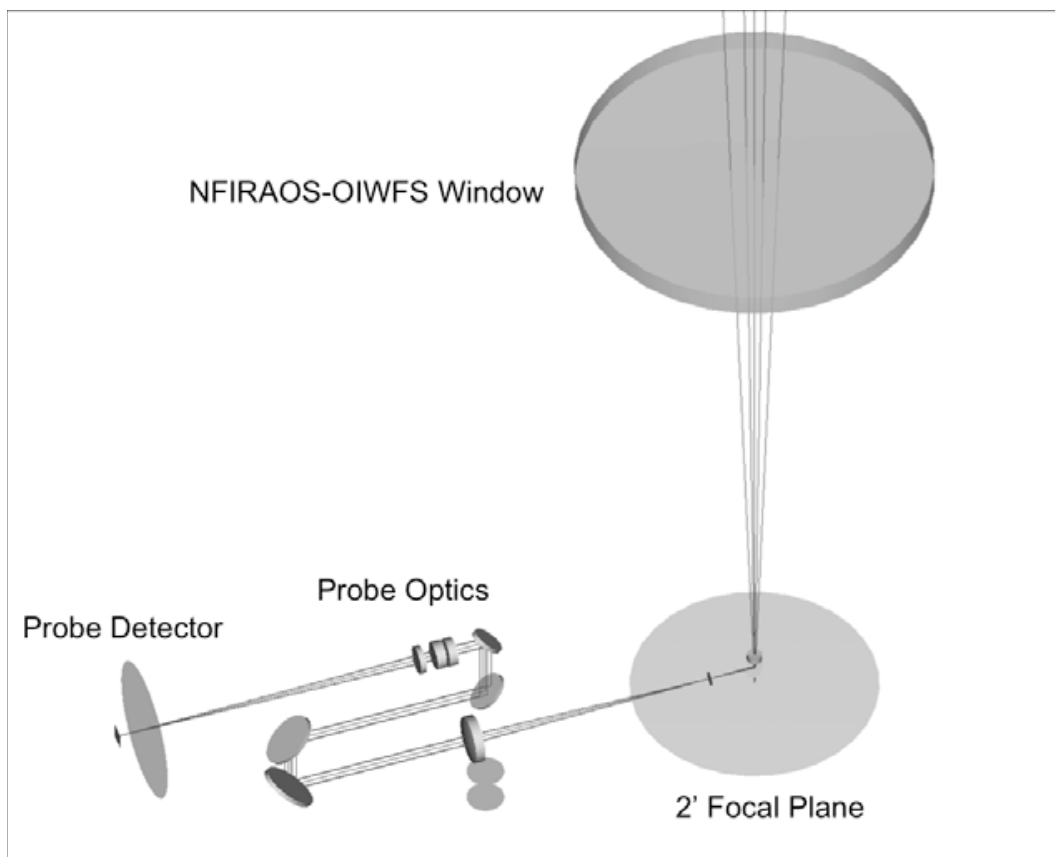

Fig. 1. General optical arrangement with respect to NFIRAOS delivered focal plane

- Robot placed pickoff and pathlength mirrors (EAGLE concept)

- Tip/tilt mirror tiling of focal plane (TIPI concept)

- Theta-Phi probes, 2 rotation stages (GMOS and Flamingos-2 OIWFS)

- Theta-R probes, 1 rotation +1 linear stages (IRMOS, KMOS)

The requirements for positioning accuracy, dithering, and non-sidereal tracking pushed the concept choice towards movable probes, and the theta-R probe concept was chosen over the theta-phi probe concept because of collision concerns. The probe pickoffs would be located at the NFIRAOS delivered focal plane approximately $750 \mathrm{~mm}$ from the NFIRAOS-IRIS mechanical interface with the theta-R probes patrolling the 2 arcmin curved focal plane field of regard. This arrangement is acceptable to the IRIS design team, with their first optic to be located approximately $100 \mathrm{~mm}$ after the delivered focal plane. Figure 1 shows this general arrangement with NFIRAOS-OIWFS window, the delivered 2 arcmin focal plane, and a single theta- $\mathrm{R}$ probe. The plate scale at the delivered focal plane is 2.18 $\mathrm{mm} / \mathrm{arcsec}$, which translates the 2 mas positioning accuracy requirement into approximately $4 \mathrm{um}$ accuracy.

With arbitrary location of the tip/tilt (TT) and tip/tilt/focus (TTF) natural guide stars it initially looked like we would need have separate horizontal patrol planes so the TT and TTF probes could reach past each other. A breakthrough came with the realization that we could configure each probe for either TT or TTF function by inserting either an imager mono lens or a $2 \times 2$ lenslet and adjusting the scale with the probe trombone mirror arrangement. This allowed us to have three identical theta- $R$ probes at 120 degree spacing around the 2 arcmin field of regard.

When this concept was analyzed for sky coverage a further breakthrough came with the realization that each probe only needs to reach 50 percent across the 2 arcmin field of regard to get a suitable natural guide star asterism around the on-axis science field. Figure 2 shows a plan view of the patrol geometry for this arrangement.

\section{Probe Design}

The theta-R probe optics design was developed to reflect the changeable TT and TTF functionality, as illustrated in figure 3 . The probe pickoff tip incorporates a converter lens, pickoff fold mirror, and 


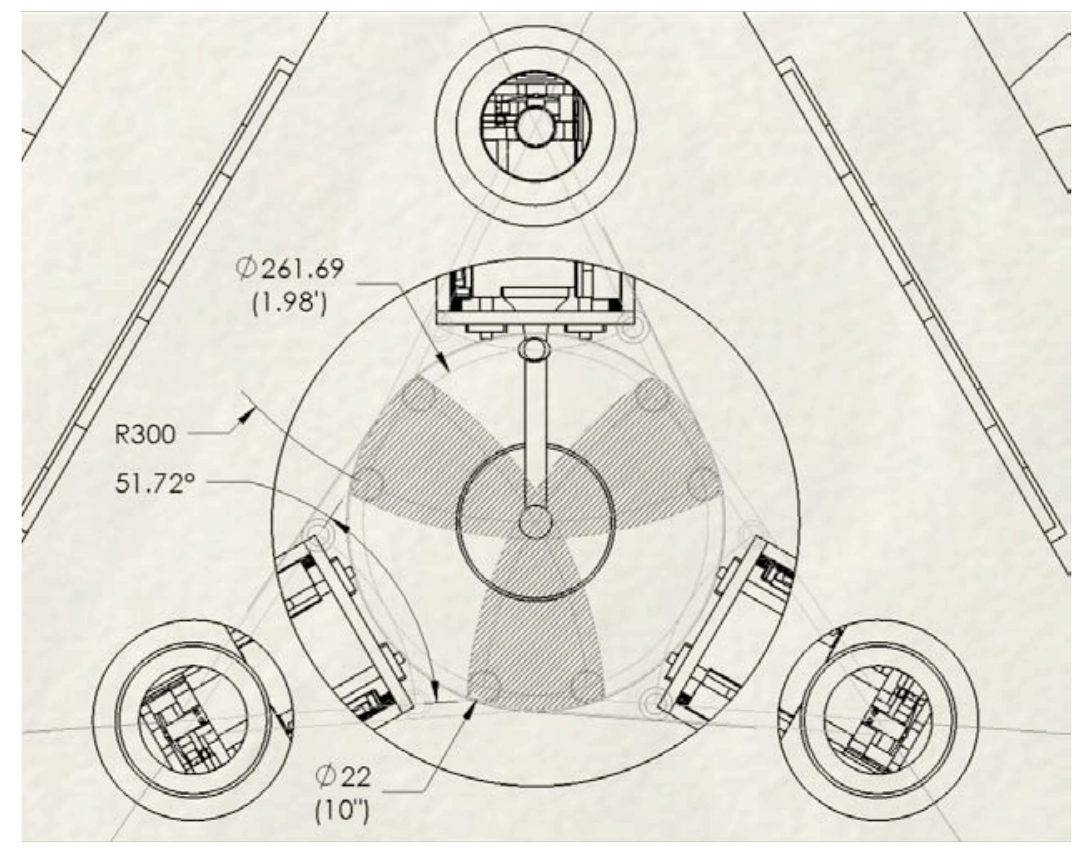

Fig. 2. Plan view of theta-R probe geometry

circular field stop sized to fit within the detector pixel area. The collimator lens on the probe arm incorporates $3 \mathrm{~mm}$ of focus motion to compensate for defocus of the curved NFIRAOS delivered focal surface and maintain probe image quality. The trombone mirrors at the back of the linear stage are adjustable to maintain the pupil patch focus for TT and TTF configurations. The trombone mirrors move at half the rate of the probe tip to maintain path length, but the TT and TTF function change necessitates separate linear actuators for the probe arm and trombone mirrors rather than a 2 to 1 screw pitch arrangement. A pair of fold mirrors translate the optical path through the rotation stage, with the remainder of the path and detector held stationary. Each probe includes a separate atmospheric dispersion compensator (ADC) to compensate for telescope azimuth change. The TT imager mono lens and the TTF $2 \times 2$ lenslet array are inserted or removed as required.

The theta- $R$ probe mechanics design was developed to support the optics design and meet the performance and accuracy requirements, as shown in figure 4.

\section{Probe Detectors}

The OIWFS theta-R probe detectors are critical to the acquisition and sky coverage performance of the combined NFIRAOS-IRIS system. The science requirement is for better than 50 percent sky coverage at the Galactic pole (sparse area of the sky) which drives us to be able to sense very faint infrared natural guide stars. We undertook a trade study of existing infrared detectors and quickly down selected to the Teledyne Hawaii H1RG detector.

At the start of each observation we need to acquire the three natural guide stars by positioning the OIWFS probes and integrating the jittering guide star images with a relatively large field of view until the AO system can close the control loop and further sharpen the image. The acquisition jitter and the acquisition probability drives our pixel count. We have chosen our probe pixel scales so that the detectors can support interchangeable TT or TTF functions; TT pixel scale $=$ lambdaH/2D $=5.67$ mas/pixel, TTF pixel scale $=$ lambdaH/D $=11.34$ mas/pixel. At these pixel scales, analysis shows that we need a minimum of 180x180 pixels to cover the worst case acquisition jitter. From an acquisition probability perspective it would be better to have more pixels, and we have settled on a baseline of $1024 \times 1024$, but this is fairly arbitrary. A compromise would be to have an infrared acquisition 


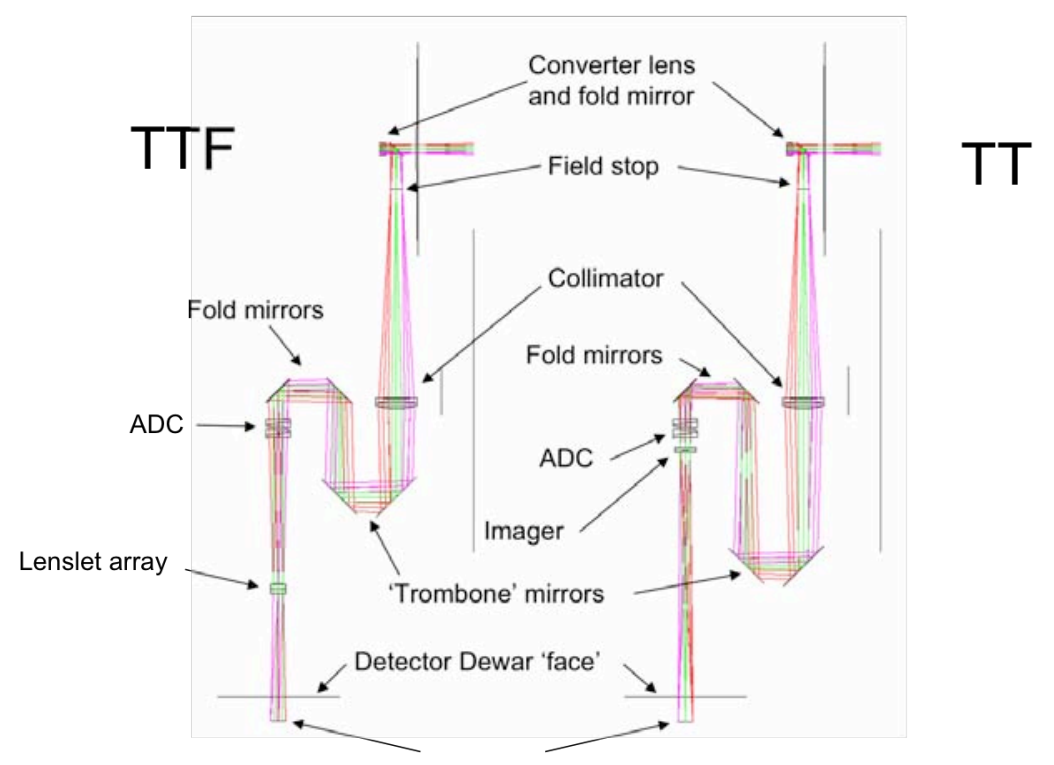

Fig. 3. Theta-R probe optics design with TT and TTF function change

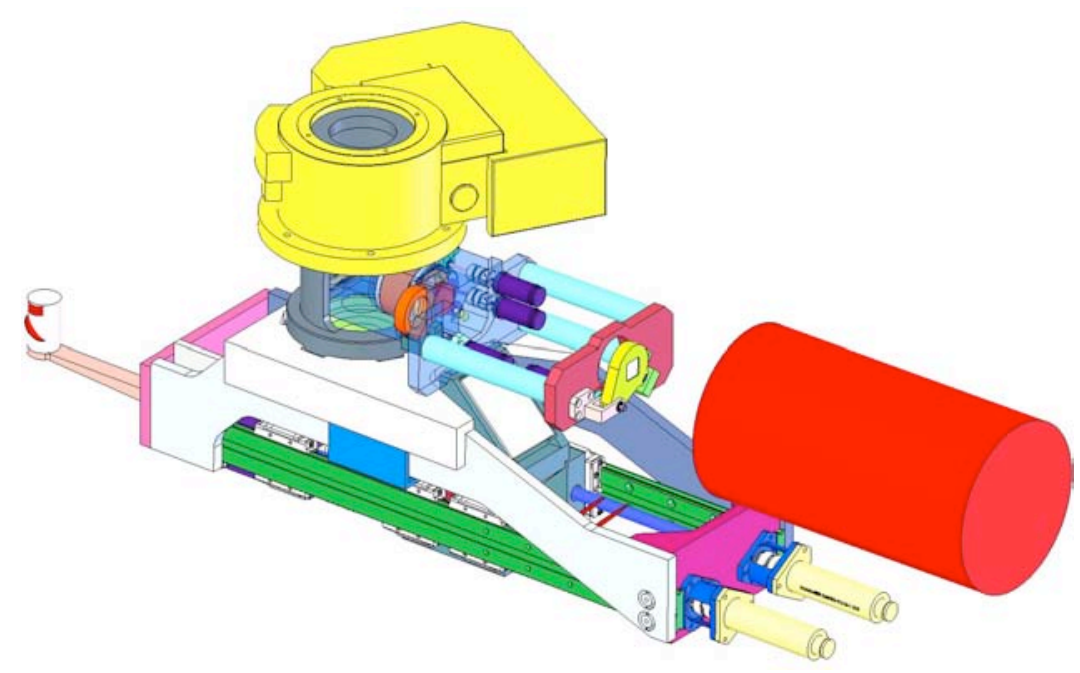

Fig. 4. Theta-R probe mechanics design

camera with lots of pixels to resolve the actual position of the natural guide stars for the probes during acquisition, so smaller detector formats could also be workable.

During acquisition the frame rate can be as slow as a few $\mathrm{Hz}$, but during observation we need to increase the frame rate up to 100 to $800 \mathrm{~Hz}$ to satisfy the AO performance goals. Currently we envisage a narrowing region-of-interest window where we read a small 10x10 pixel window at higher frame rate. This windowing should be able to have arbitrary position within the array, to help with all three probes acquiring. The minimum size of the pixel window is driven by the read noise to be smaller (multiple fast sampling techniques to drive down the read noise) and by the ability to maintain lock to be larger (things that go bump in the night and move the image). Note that this windowing approach is predicated by current Teledyne HxRG ROIC capabilities, but alternative detectors may have different modalities to solve the frame rate versus read noise underlying requirements. 


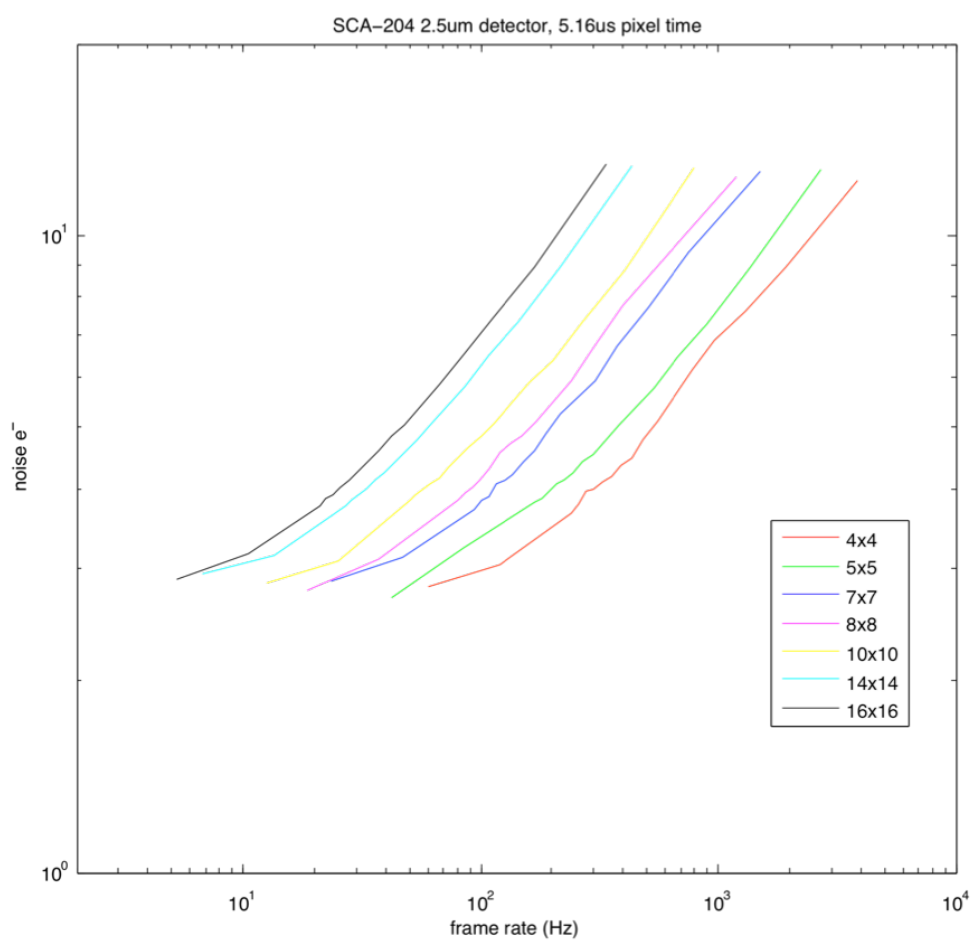

Fig. 5. Preliminary results - HxRG detector read noise vs. frame rate for window sizes

The sky coverage requirement drives the OIWFS detector quantum efficiency and read noise to allow us to acquire and image very faint stars at the high frame rate. Currently we are specifying 60-80 percent quantum efficiency and 3-5 electrons read noise over a passband from 0.8 to $1.7 \mathrm{um}$. We have developed a sky coverage modeling tool based on existing star catalogues that we use to explore the trade space of these variables. We have received a sample of the H2RG with an improved low noise material and are conducting tests. Early results are promising and 3 e- read noise at small window sizes looks attainable.

\section{Macro Mechanics}

We have developed concept designs for the macro-mechanics in conjunction with the IRIS design team including,

- the fixed NFIRAOS kinematic interface plate and thermal seal

- instrument rotator bearing

- instrument rotator gear ring and motors(2)

- the support frame between the rotator and the IRIS interface plate

- the OIWFS thermal structure

- the OIWFS support frame and platform

- the IRIS kinematic interface plate

- and the service wrap

An exploded view of these elements is shown in figure 5. 
First conference on Adaptive Optics for Extremely Large Telescopes

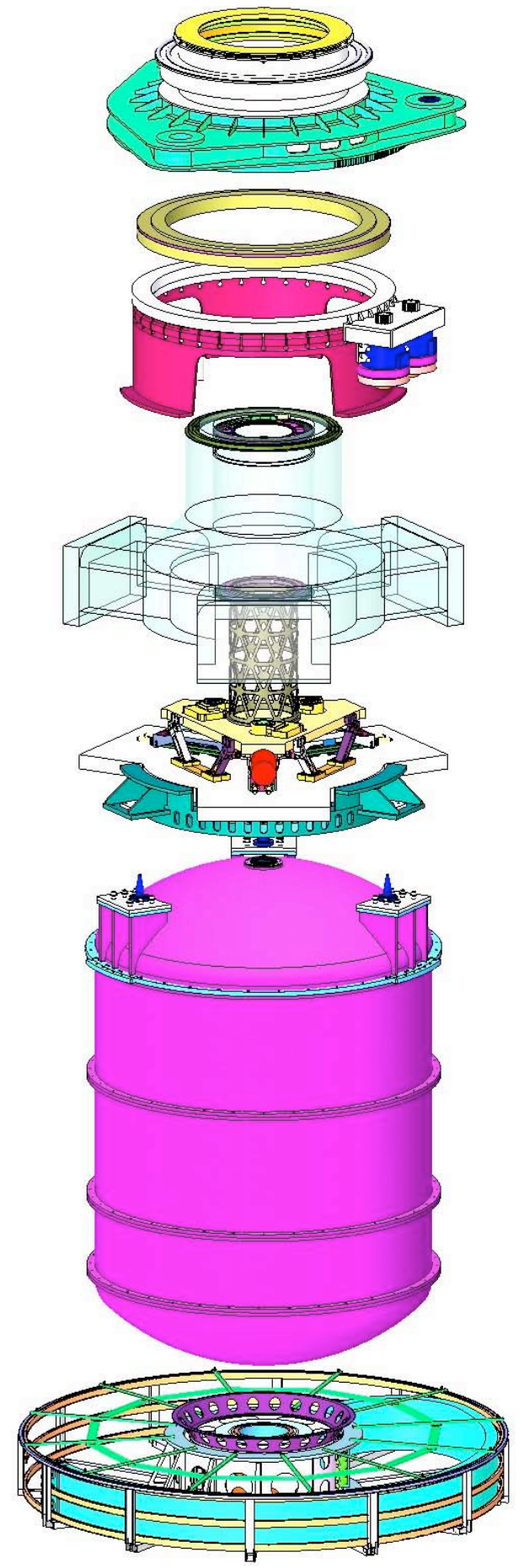

Fig. 6. Exploded view of macro mechanics 